\title{
Modulation instabilities in two-core optical fibers
}

\author{
Jin Hua Li, ${ }^{1}$ Kin Seng Chiang, ${ }^{2, *}$ and Kwok Wing Chow ${ }^{1}$ \\ ${ }^{1}$ Department of Mechanical Engineering, University of Hong Kong, Hong Kong SAR, China \\ ${ }^{2}$ Department of Electronic Engineering, City University of Hong Kong, Hong Kong SAR, China \\ ${ }^{*}$ Corresponding author: eeksc@cityu.edu.hk
}

Received January 26, 2011; revised April 18, 2011; accepted May 20, 2011;

posted May 20, 2011 (Doc. ID 141244); published June 16, 2011

\begin{abstract}
Modulation instability (MI) of cw states of a two-core fiber, incorporating the effects of coupling-coefficient dispersion (CCD), is studied by solving a pair of generalized, linearly coupled nonlinear Schrödinger equations. CCD refers to the property that the coupling coefficient depends on the optical wavelength, and earlier studies of MI do not account for this physics. CCD does not seriously affect the symmetric/antisymmetric cw, but can drastically modify the MI of the asymmetric state. Generally, new MI frequency bands are produced, and CCD reduces (enhances) the original MI band in the anomalous (normal) dispersion regime. Another remarkable result is the existence of a critical value for the CCD, where the MI gain spectrum undergoes an abrupt change. In the anomalous dispersion regime, a new low-frequency MI band is generated. In the normal dispersion regime, an MI band vanishes, reappears, and then moves up in frequency on crossing this critical value. In both dispersion regimes, the relative magnitude of the low-frequency band and the high-frequency band depends strongly on the total input power. It is possible to switch the dominant MI frequency between a low frequency and a high frequency by tuning the total input power, providing a promising scheme to manipulate MI-related nonlinear effects in two-core fibers. The MI bands are independent of the third-order dispersion, but can be shifted significantly by self-steepening at a sufficiently high total input power. The evolution of MI from a cw input is also demonstrated with a wave propagation study. (c) 2011 Optical Society of America
\end{abstract}

OCIS codes: $\quad 060.1810,060.4080,060.4370,190.3100,190.4370$.

\section{INTRODUCTION}

Modulation instability (MI) is the process where weak perturbations imposed on a cw state grow exponentially, as a result of the interplay between nonlinear and dispersive effects [1-18]. MI has been studied since the 1960s in many fields of science and engineering. In the optics community, MI is relevant in many topics, including Bragg gratings [트, 2 , cross-phase modulations [3,4] , four-wave mixing [5] , novel materials [6,7], parametric oscillators [8], polarization and birefringence [9-12], saturable nonlinearity [13], spatial instability [14], supercontinuum generation [ $[\overline{15}]$, and temporal solitons in fibers [ $[16-18]$. The occurrence of MI is closely related to the classical physical phenomenon of Fermi-PastaUlam recurrence $[19,20]$, and may lead to the formation of solitons [21].

For temporal pulse propagation in a single-mode optical fiber, governed by the nonlinear Schrödinger equation, the relevant factors are cubic (Kerr) nonlinearity and group velocity dispersion (GVD). MI then occurs in the anomalous dispersion regime [16]. With additional physics, MI can also arise in other settings, e.g., in the normal dispersion regime by cross-phase modulation [3], in the presence of higher even-order dispersions [17] and loss dispersion [18]. In this paper, we study MI in a two-core optical fiber in both the anomalous and normal dispersion regimes, incorporating the effects of couplingcoefficient dispersion (CCD).

In a two-core fiber, optical power can be transferred between the two cores periodically [22]. This phenomenon plays an important role in many modern optical devices. Theoretically, the evolution of the electric-field envelopes along the fiber is governed by a system of linearly coupled nonlinear Schrödinger equations. The coefficient of the linear coupling, known as the coupling coefficient, dictates the strength of the power transfer, and its magnitude depends on the design and the operation condition of the fiber.

In general, the coupling coefficient depends on the optical wavelength, which is the physical principle of wavelength filters formed with two-core fibers demonstrated a long time ago [23]. The effects of a dispersive coupling coefficient on the propagation of pulses in a two-core fiber, however, have not been recognized until recently [24-31]. CCD in a two-core fiber can lead to significant pulse distortion or even pulse breakup, and thus seriously affect nonlinear pulse switch-

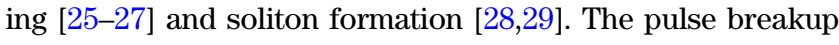
effect in a two-core fiber has been observed experimentally [30] and applied to the generation of high-speed pulse trains [31].

While MI in a two-core fiber has been analyzed previously $[32,33]$, the effects of CCD have not been considered. The primary objective of the present study is to show how CCD affects MI in a two-core fiber.

Two general cw states must be considered:

- the symmetric/antisymmetric state, where the optical powers in the two cores are always equal (the state is symmetric or antisymmetric depending on whether the waves in the two cores are in phase or completely out of phase respectively);

- the asymmetric state, where the optical powers in the two cores are unequal.

MI for both the symmetric/antisymmetric [32] and asymmetric [33] states has been analyzed earlier, but without taking CCD into account. 
In this paper, linear stability calculations reveal that CCD does not seriously affect the MI of the symmetric/ antisymmetric cw state, but can drastically modify the characteristics of the MI associated with the asymmetric state. The main results are:

a. The presence of CCD generally reduces (enhances) the MI of the two-core fiber in the anomalous (normal) dispersion regime.

b. The actual maximum growth rates for MI with CCD can be substantially different from those without CCD.

c. The original MI branches into two frequency bands. Their relative magnitude depends on the input power level, which provides a potentially promising way to manipulate light signals in fibers, by switching the dominant MI frequency with a tunable total input power.

d. A critical value of CCD exists, where the MI properties change abruptly. For anomalous GVD, a new MI band appears around this critical value. For normal GVD, disappearance and reappearance of a MI band can be observed.

e. The third-order dispersion has no effect on the MI gain spectrum, while self-steepening (SS) can modify the MI gain spectrum significantly at a sufficiently high total input power.

The MI analysis is also verified with a wave propagation study that demonstrates the evolution of a modulated wave from a cw input.

\section{COUPLED-MODE EQUATIONS}

We consider a two-core fiber, where each core supports only a single mode. The evolution of the electric-field envelopes along the fiber is described by a pair of generalized, linearly coupled nonlinear Schrödinger equations [24-26]

$i \frac{\partial a_{1}}{\partial z}-\frac{1}{2} \beta_{2} \frac{\partial^{2} a_{1}}{\partial t^{2}}+\gamma\left|a_{1}\right|^{2} a_{1}+C a_{2}+i C_{1} \frac{\partial a_{2}}{\partial t}=0$,

$i \frac{\partial a_{2}}{\partial z}-\frac{1}{2} \beta_{2} \frac{\partial^{2} a_{2}}{\partial t^{2}}+\gamma\left|a_{2}\right|^{2} a_{2}+C a_{1}+i C_{1} \frac{\partial a_{1}}{\partial t}=0$,

where $a_{1}$ and $a_{2}$ are the slowly varying electric-field envelopes in the two cores; $z$ and $t$ are the propagation distance and the retarded time coordinate respectively; $\beta_{2}$ measures the GVD at the carrier frequency $\left(\beta_{2}<0\right.$ for anomalous dispersion and $\beta_{2}>0$ for normal dispersion); $\gamma$ is the self-phase modulation (SPM) parameter with $\gamma=2 \pi n_{2} /\left(\lambda A_{\text {eff }}\right)$, where $\lambda, n_{2}$, and $A_{\text {eff }}$ are the free-space optical wavelength, nonlinear refractive index of the fiber material, and the effective area of each core, respectively; $C$ is the coupling coefficient, which is proportional to the spatial overlap between the mode fields in the two cores and is responsible for the periodic power exchange between the two cores [22]; $C_{1}=d C / d \omega$ (where $\omega$ denotes the angular optical frequency) represents the CCD at the carrier frequency $[24,25]$, which is equivalent to the intermodal dispersion arising from the group-delay difference between the even and odd supermodes of the two-core fiber.

For a typical two-core fiber, we have $1<\gamma<10 /(\mathrm{kW} \cdot \mathrm{m})$, $0<C<1000 / \mathrm{m}$, and $0>C_{1}>-10 \mathrm{ps} / \mathrm{m}[24,34]$. The values of $C$ and $C_{1}$ can be made arbitrarily small by increasing the core separation. It is possible, however, to achieve $C_{1}=0$ without reducing $C$, by using a special fiber design [34]. Recently, a photonic bandgap two-core fiber that gives $C=0$ has been demonstrated [35]. Nevertheless, such a decoupled twocore fiber can have an appreciable value of $C_{1}$ (around $-1 \mathrm{ps} / \mathrm{m}$ ), while maintaining $C=0$ [36].

Equation (1) admits both symmetric/antisymmetric and asymmetric $\mathrm{cw}$ solutions. Previous studies of $\mathrm{MI}$ in a two-core fiber $[\underline{32}, \underline{33}]$ do not account for the effects of the $C_{1}$ terms, and our goal is to perform such an investigation.

\section{SYMMETRIC/ANTISYMMETRIC SOLUTIONS}

A symmetric/antisymmetric cw solution of Eq. (1) is

$$
a_{1}=\sqrt{P_{0}} \exp (i k z), \quad a_{2}=\delta \sqrt{P_{0}} \exp (i k z),
$$

with $k=\gamma P_{0}+\delta C$, and $\delta= \pm 1$ with the positive and negative signs corresponding to the symmetric and antisymmetric solutions, respectively. To study the stability of the solutions, we put

$$
\begin{aligned}
& a_{1}=\left(\sqrt{P_{0}}+u\right) \exp (i k z) \\
& a_{2}=\left(\delta \sqrt{P_{0}}+v\right) \exp (i k z)
\end{aligned}
$$

where $u$ and $v$ represent weak perturbations in the two cores. On substituting Eq. (ㅁ) into Eq. (1), linearization gives

$$
\begin{aligned}
& i \frac{\partial u}{\partial z}-\frac{1}{2} \beta_{2} \frac{\partial^{2} u}{\partial t^{2}}+\left[\left(\gamma P_{0}-\delta C\right) u+\gamma P_{0} u^{*}\right]+C v+i C_{1} \frac{\partial v}{\partial t}=0 \\
& i \frac{\partial v}{\partial z}-\frac{1}{2} \beta_{2} \frac{\partial^{2} v}{\partial t^{2}}+\left[\left(\gamma P_{0}-\delta C\right) v+\gamma P_{0} v^{*}\right]+C u+i C_{1} \frac{\partial u}{\partial t}=0
\end{aligned}
$$

We now search for sidebands in the following form:

$$
\begin{aligned}
& u=F_{1} \exp (i K z-i \Omega t)+G_{1} \exp (-i K z+i \Omega t), \\
& v=F_{2} \exp (i K z-i \Omega t)+G_{2} \exp (-i K z+i \Omega t),
\end{aligned}
$$

where $F_{1}, G_{1}, F_{2}$, and $G_{2}$ are real, and $K$ and $\Omega$ are the wavenumber and the modulation frequency, respectively. Insisting on nontrivial solutions of $F_{1}, G_{1}, F_{2}$, and $G_{2}$ leads to the dispersion relation

$$
\begin{aligned}
& {\left[\left(K-\delta C_{1} \Omega\right)^{2}-r_{1}\right]\left[\left(K+\delta C_{1} \Omega\right)^{2}-r_{2}\right]=0,} \\
& r_{1}=\frac{1}{4} \beta_{2} \Omega^{2}\left(\beta_{2} \Omega^{2}+4 \gamma P_{0}\right), \\
& r_{2}=\frac{1}{4}\left(\beta_{2} \Omega^{2}-4 \delta C\right)\left(\beta_{2} \Omega^{2}-4 \delta C+4 \gamma P_{0}\right) .
\end{aligned}
$$

MI occurs when $K$ is complex for real $\Omega$, i.e., when the weak perturbations grow along the fiber with a gain given by $\operatorname{Im}(K)$. The necessary condition for Eq. (ㅁ) is

$$
r_{1}<0 \quad \text { or } \quad r_{2}<0 \text {. }
$$

The MI that corresponds to a negative $r_{1}$ occurs only in the anomalous dispersion regime. The MI that corresponds to a negative $r_{2}$ can occur in both the anomalous and normal dispersion regimes, and arises from the presence of the coupling 
coefficient $C$. The analysis is mathematically identical to that obtained previously $[32,33]$. In essence, CCD just shifts the range of the instability frequency and does not change the growth rate of the MI. Physically, the symmetric (or antisymmetric) wave is carried only by the even (or odd) supermode of the two-core fiber. As only one supermode is present, intermodal dispersion does not play a significant role here [24].

\section{ASYMMETRIC SOLUTIONS}

Equation (1) also admits asymmetric cw solutions

$$
a_{1}=\sqrt{P_{1}} \exp (i k z), \quad a_{2}=\sqrt{P_{2}} \exp (i k z),
$$

with $P_{2}=C^{2} /\left(\gamma^{2} P_{1}\right)$ and $k=\gamma P=\gamma\left(P_{1}+P_{2}\right)$, where $P=$ $P_{1}+P_{2}$ is the total power launched into the fiber. There are two observations here:

- For a fiber with a given $C$, a minimum total power, $P_{\text {min }}=2 C / \gamma$, is necessary to sustain this cw, and at this special value $\left(P=P_{\min }\right), P_{1}=P_{2}=C / \gamma$ gives a symmetric cw state.

- $\quad$ For $P>P_{\text {min }}, P_{1} \neq P_{2}$, and an asymmetric state is generated. The power distribution in the two cores is given by

$$
\left(P_{1}, P_{2}\right)=\left(\frac{1}{2} P \pm \frac{1}{2} \sqrt{P^{2}-P_{\min }^{2}}, \frac{1}{2} P \mp \frac{1}{2} \sqrt{P^{2}-P_{\text {min }}^{2}}\right) .
$$

Thus, for an asymmetric cw, the difference between the powers in the two cores becomes larger if the total power increases.

For MI, the same theoretical techniques, as detailed in Section 3 , generate the dispersion relation

$$
\begin{aligned}
& {\left[\left(K+\frac{\sqrt{2}}{2} \Omega C_{1}\right)^{2}-s_{1}\right]\left[\left(K-\frac{\sqrt{2}}{2} \Omega C_{1}\right)^{2}-s_{2}\right]=s,} \\
& s_{1}=\frac{1}{2} \gamma^{2} P^{2}-2 C^{2}+\frac{1}{4} \beta_{2}^{2} \Omega^{4}+\left(\frac{1}{2} C_{1}^{2}-\sqrt{2} \beta_{2} C\right) \Omega^{2}, \\
& s_{2}=\frac{1}{2} \gamma^{2} P^{2}-2 C^{2}+\frac{1}{4} \beta_{2}^{2} \Omega^{4}+\left(\frac{1}{2} C_{1}^{2}+\sqrt{2} \beta_{2} C\right) \Omega^{2}, \\
& s=\frac{1}{2} \beta_{2}^{2} C_{1}^{2} \Omega^{6}-\left(5 \beta_{2}^{2} C^{2}+C_{1}^{4}-\beta_{2}^{2} \gamma^{2} P^{2}\right) \Omega^{4} \\
& \quad-\left(\gamma^{2} P^{2}-4 C^{2}\right)\left(\beta_{2} \gamma P+2 C_{1}^{2}\right) \Omega^{2}+\frac{1}{4}\left(\gamma^{2} P^{2}-4 C^{2}\right)^{2} .
\end{aligned}
$$

Again, MI occurs when $K$ is complex for real $\Omega$, and the gain is then

$$
g(\Omega)=\operatorname{Im}(K)
$$

At the minimum power $P_{\min }=2 C / \gamma$, Eq. (11) reduces to Eq. (6), consistent with the fact that the asymmetric state degenerates to the symmetric state at that point, as $P_{1}=P_{2}=$ $P_{\min } / 2$ there.
As the dispersion relation is even, i.e., $g(\Omega)=g(-\Omega)$, it is sufficient to show the spectrum for a positive $\Omega$. The numerical results are presented in Sections $\underline{4 . A}$ and $\underline{4}$.B.

\section{A. Anomalous Dispersion Regime}

As an illustrative example, we select $\beta_{2}=-0.02 \mathrm{ps}^{2} / \mathrm{m}$ and $\gamma=2.5 /(\mathrm{kW} \cdot \mathrm{m})$. It is instructive to establish a benchmark by examining the variation of MI with the total power $P$ and the coupling coefficient $C$ in the absence of CCD (i.e., $\left.C_{1}=0\right)$.

a. For a typical value of $C=200 / \mathrm{m}$, there is a single MI band, with the maximum gain increasing with larger total input power $P$, as shown in Fig. 1 .

b. For a typical value of $P=\overline{2} 00 \mathrm{~kW}$, there is again a single MI band, but now the maximum gain decreases with larger values of $C$, as shown in Fig. 2. At $C=0$, the two cores are actually uncoupled. Consequently, any power transfer between the two cores, i.e., a nonvanishing $C$, always lowers the MI gain.

We next concentrate on the effects of $C_{1}$ by fixing $C=200 / \mathrm{m}$, which corresponds to $P_{\min }=160 \mathrm{~kW}$. Figure $\underline{3}$ shows the variation of the MI gain spectrum $g(\Omega)$ with $C_{1}$ at a total power of $P=170 \mathrm{~kW}$, which is slightly larger than $P_{\text {min }}$. Around $C_{1}=0$, a single low-frequency band exists, with maximum gain rate decreasing with increasing $\left|C_{1}\right|$.

As $C_{1}$ reaches a critical value, termed $C_{1 \mathrm{cr}}$ here (at around $-2 \mathrm{ps} / \mathrm{m}$ for this example), the instability band shifts gradually toward the high frequency and eventually becomes a weak, narrow high-frequency band. On the other hand, a new low-frequency band is generated quickly as $\left|C_{1}\right|$ crosses the critical value, and the gain of this band is insensitive to any

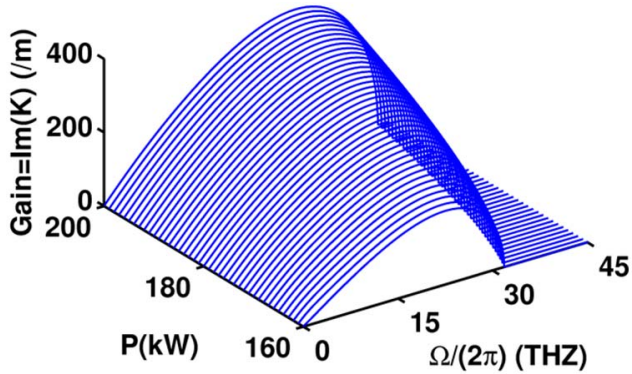

(a)

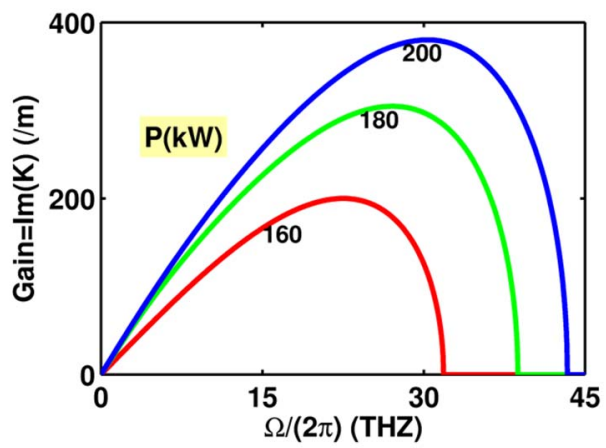

(b)

Fig. 1. (Color online) (a) Three-dimensional (3D) and (b) twodimensional (2D) plots showing the variation of the MI gain spectrum $g(\Omega)$ with the total power $P$, calculated for the anomalous dispersion regime with $\beta_{2}=-0.02 \mathrm{ps}^{2} / \mathrm{m}, \gamma=2.5 /(\mathrm{kW} \cdot \mathrm{m}), C=200 / \mathrm{m}$, and $C_{1}=0$. 


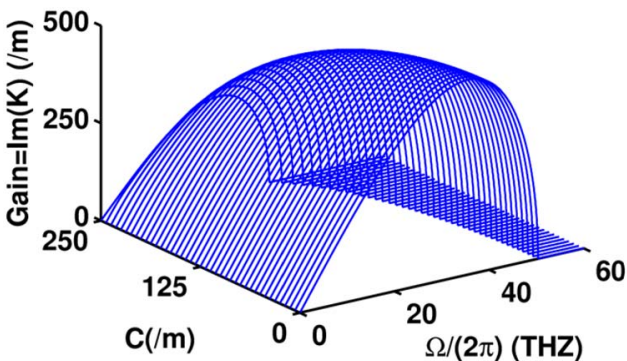

(a)

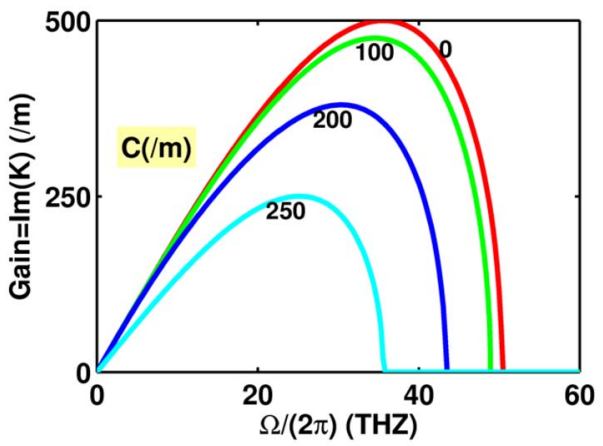

(b)

Fig. 2. (Color online) (a) 3D and (b) 2D plots showing the variation of the MI gain spectrum $g(\Omega)$ with the coupling coefficient $C$, calculated for the anomalous dispersion regime with $\beta_{2}=-0.02 \mathrm{ps}^{2} / \mathrm{m}$, $\gamma=2.5 /(\mathrm{kW} \cdot \mathrm{m}), C_{1}=0$, and $P=200 \mathrm{~kW}$.

further increase in $\left|C_{1}\right|$. These new features are purely caused by CCD, and have not been studied previously.

Figure 4 shows the dependence of the MI gain spectrum $g(\Omega)$ on $C_{1}$ at a total power of $P=400 \mathrm{~kW}$, which is much larger than $P_{\min }$. While the patterns in Figs. $\underline{3}$ and $\underline{4}$ are similar, significant differences exist:

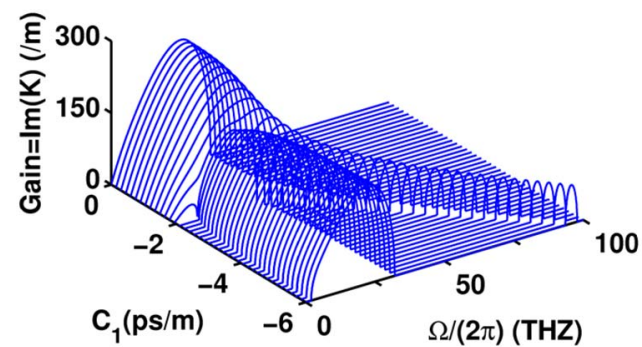

(a)

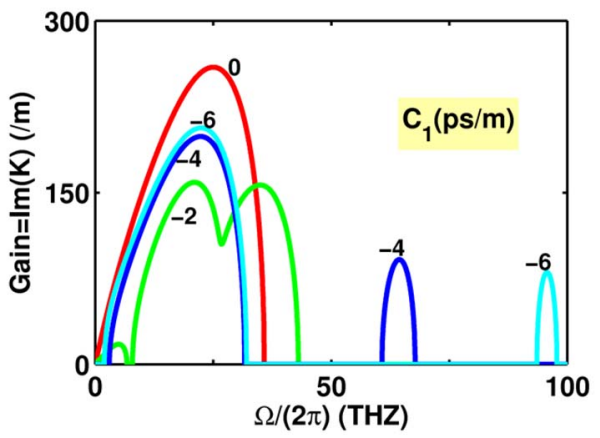

(b)

Fig. 3. (Color online) (a) 3D and (b) 2D plots showing the variation of the MI gain spectrum $g(\Omega)$ with the coupling-coefficient parameter $C_{1}$, calculated for the anomalous dispersion regime with $\beta_{2}=-0.02 \mathrm{ps}^{2} / \mathrm{m}, \gamma=2.5 /(\mathrm{kW} \cdot \mathrm{m}), C=200 / \mathrm{m}$, and $P=170 \mathrm{~kW}$.

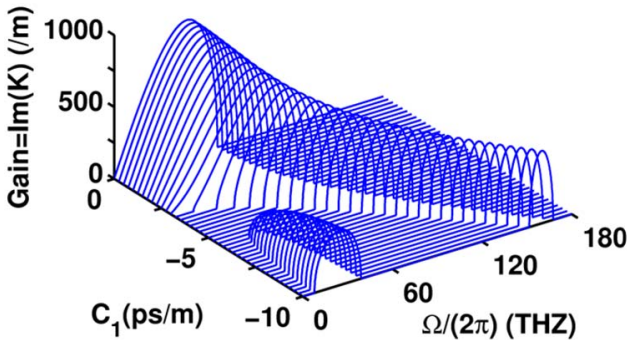

(a)

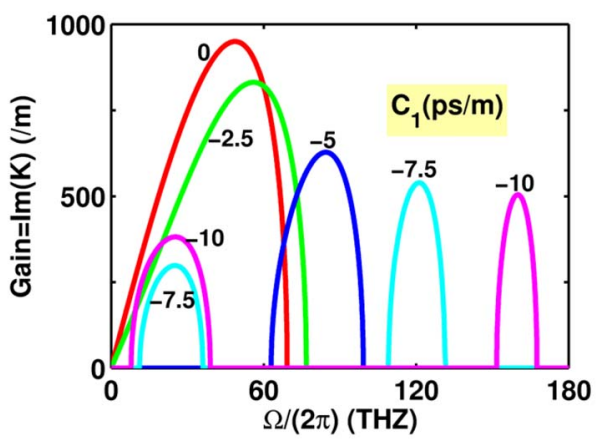

(b)

Fig. 4. (Color online) (a) 3D and (b) 2D plots showing the variation of the MI gain spectrum $g(\Omega)$ with the coupling-coefficient dispersion parameter $C_{1}$, calculated for the anomalous dispersion regime with $\beta_{2}=-0.02 \mathrm{ps}^{2} / \mathrm{m}, \gamma=2.5 /(\mathrm{kW} \cdot \mathrm{m}), C=200 / \mathrm{m}$, and $P=400 \mathrm{~kW}$.

a. a much larger critical value $C_{1 \mathrm{cr}}$ (at around $-5.4 \mathrm{ps} / \mathrm{m}$ ) for the present case of higher total power

b. a more pronounced gain in the high-frequency band, as the low-frequency band weakens

These results suggest the possibility of switching the dominant MI from a low-frequency band to a high-frequency band by increasing the total input power $P$, as long as the value of $\left|C_{1}\right|$ is sufficiently large.

The magnitude of $C_{1 \mathrm{cr}},\left|C_{1 \mathrm{cr}}\right|$, increases with $P, \gamma$, and $\left|\beta_{2}\right|$, but decreases with an increase in $C$, as shown in Fig. $\underline{5}$.

\section{B. Normal Dispersion Regime}

As an illustrative example, we take $\beta_{2}=0.02 \mathrm{ps}^{2} / \mathrm{m}$, which can be achieved by operating the fiber at a sufficiently short wavelength. Accordingly, the value of $\gamma$ is adjusted to $5.0 /(\mathrm{kW} \cdot \mathrm{m})$. Again, we first set $C_{1}=0$, and study the dependence of MI on $P$ (total power) and $C$ (coupling coefficient) as background information.

For a typical value $C=200 / \mathrm{m}$, there is a single MI band at $P=P_{\text {min }}=80 \mathrm{~kW}$, as shown in Fig. $\underline{6}$. As $P$ increases slightly, a new MI band on the low-frequency side is generated. Both the original band and the new band shift toward the highfrequency range as $P$ increases. The gain of the low-frequency band increases rapidly initially, but later becomes saturated. The gain of the high-frequency band decreases as $P$ increases. At extremely large values of $P$, the gain will eventually vanish for the following reason. According to Eq. (10), one core would then dominate the dynamics, and MI cannot exist for one core in the normal dispersion regime.

For the dependence on $C$, we choose the typical value $P=100 \mathrm{~kW}$. At $C=0$, which corresponds to a single-core fiber, there is no MI. As $C$ increases from zero, two MI bands 

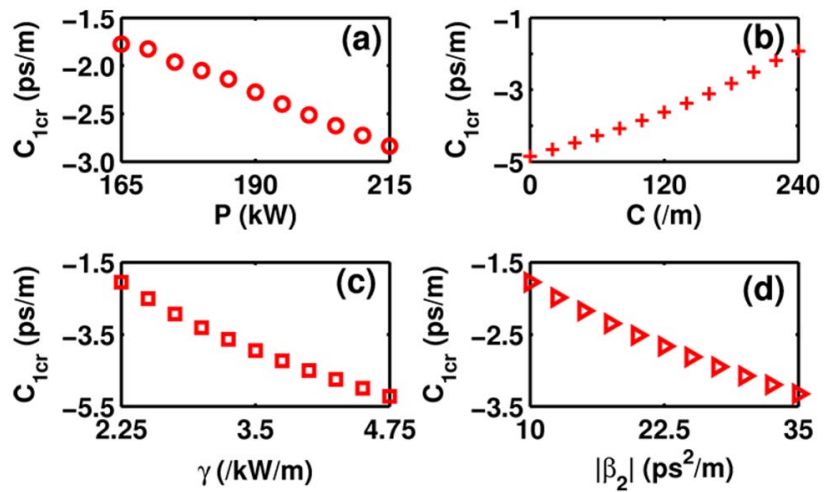

Fig. 5. (Color online) Dependence of the critical value $C_{1 \mathrm{cr}}$ on (a) total input power $P$ for $\beta_{2}=-0.02 \mathrm{ps}^{2} / \mathrm{m}, \gamma=2.5 /(\mathrm{kW} \cdot \mathrm{m})$, and $C=200 / \mathrm{m}$; (b) coupling coefficient $C$ for $\beta_{2}=-0.02 \mathrm{ps}^{2} / \mathrm{m}, \gamma=$ $2.5 /(\mathrm{kW} \cdot \mathrm{m})$, and $P=200 \mathrm{~kW}$; (c) SPM parameter $\gamma$ for $\beta_{2}=$ $-0.02 \mathrm{ps}^{2} / \mathrm{m}, C=200 / \mathrm{m}$, and $P=200 \mathrm{~kW}$; and (d) magnitude of GVD $\left|\beta_{2}\right|$ for $\gamma=2.5 /(\mathrm{kW} \cdot \mathrm{m}), C=200 / \mathrm{m}$, and $P=200 \mathrm{~kW}$.

emerge, as shown in Fig. 7 . The two MI bands grow with $C$ and eventually merge into a broad band.

We now concentrate on $C_{1}$. For the typical values $P=$ $100 \mathrm{~kW}$ (slightly larger than $P_{\min }=80 \mathrm{~kW}$ ) and $C=200 / \mathrm{m}$, there are two bands at $C_{1}=0$, as shown in Fig. 8. As $\left|C_{1}\right|$ increases from zero, the low-frequency band grows rapidly initially and then saturates, while the high-frequency band shrinks. The high-frequency band actually vanishes at a critical value of $C_{1}$, termed again $C_{1 \mathrm{cr}}$ here. As $\left|C_{1}\right|>\left|C_{1 \mathrm{cr}}\right|$, the high-frequency band reappears and grows gradually with $\left|C_{1}\right|$, shifting toward even higher frequencies.

Regarding the magnitude of the instability, the MI growth rates can be tremendously higher for a finite value of $C_{1}$, as compared with those for the case of $C_{1}=0$ (Fig. $\underline{8}$ and,

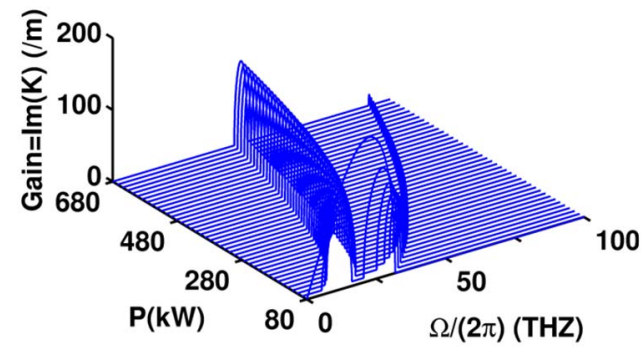

(a)

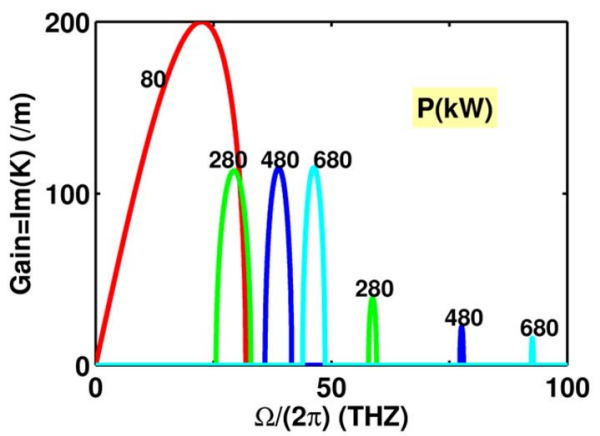

(b)

Fig. 6. (Color online) (a) 3D and (b) 2D plots showing the variation of the MI gain spectrum $g(\Omega)$ with the total power $P$, calculated for the normal dispersion regime with $\beta_{2}=0.02 \mathrm{ps}^{2} / \mathrm{m}, \gamma=5.0 /(\mathrm{kW} \cdot \mathrm{m})$, $C=200 / \mathrm{m}$, and $C_{1}=0$.

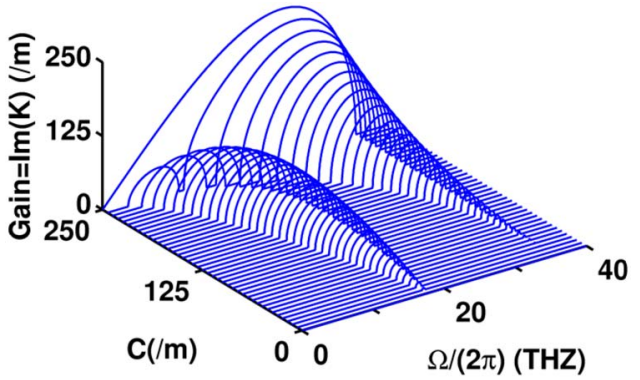

(a)

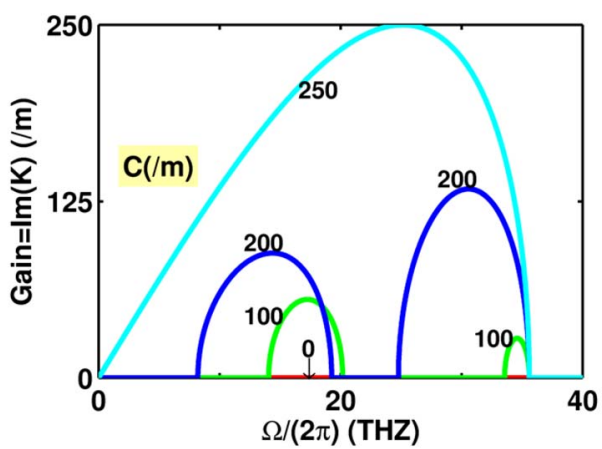

(b)

Fig. 7. (Color online) (a) 3D and (b) 2D plots showing the variation of the MI gain spectrum $g(\Omega)$ with the coupling coefficient $C$, calculated for the normal dispersion regime with $\beta_{2}=0.02 \mathrm{ps}^{2} / \mathrm{m}$, $\gamma=5.0 /(\mathrm{kW} \cdot \mathrm{m}), C_{1}=0$, and $P=100 \mathrm{~kW}$.

in a dramatic manner, Fig. 9, where a higher input power is employed).

The relative magnitude of the frequency bands depends on the power, as shown in Fig. 9. At $P=600 \mathrm{~kW}$ (much larger than $P_{\text {min }}=80 \mathrm{~kW}$ ), the critical value $C_{1 \mathrm{cr}}$ decreases to a value

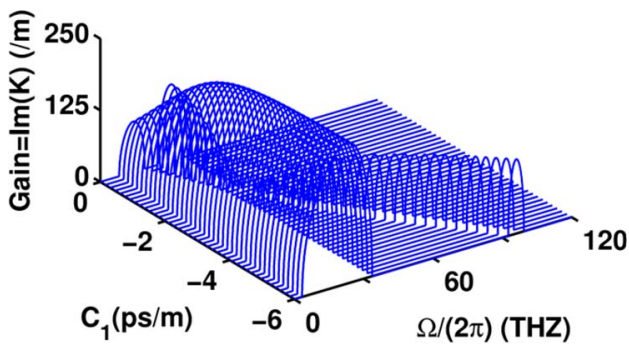

(a)

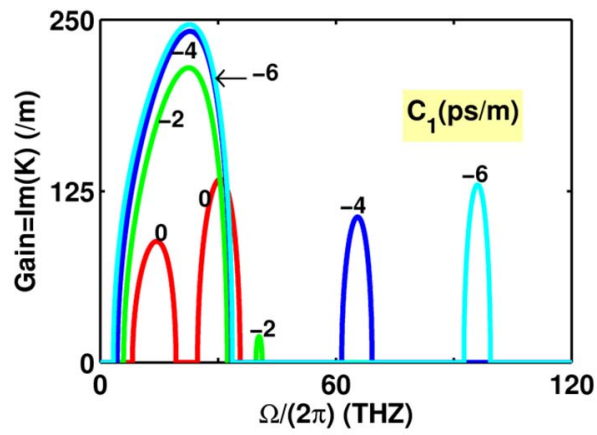

(b)

Fig. 8. (Color online) (a) 3D and (b) 2D plots showing the variation of the MI gain spectrum $g(\Omega)$ with the coupling-coefficient dispersion parameter $C_{1}$, calculated for the normal dispersion regime with $\beta_{2}=0.02 \mathrm{ps}^{2} / \mathrm{m}, \gamma=5.0 /(\mathrm{kW} \cdot \mathrm{m}), C=200 / \mathrm{m}$, and $P=100 \mathrm{~kW}$. 


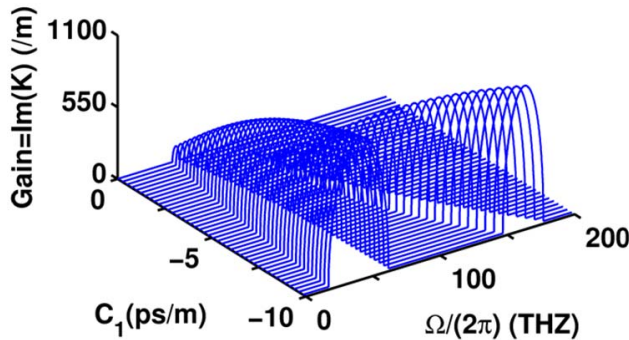

(a)

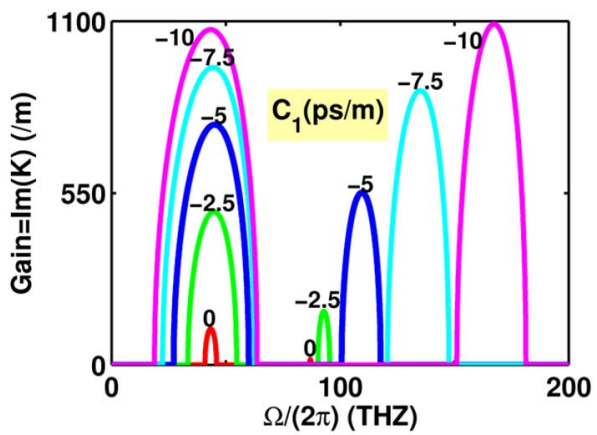

(b)

Fig. 9. (Color online) (a) 3D and (b) 2D plots showing the variation of the MI gain spectrum $g(\Omega)$ with the coupling-coefficient dispersion parameter $C_{1}$, calculated for the normal dispersion regime with $\beta_{2}=0.02 \mathrm{ps}^{2} / \mathrm{m}, \gamma=5.0 /(\mathrm{kW} \cdot \mathrm{m}), C=200 / \mathrm{m}$, and $P=600 \mathrm{~kW}$.

around $-0.7 \mathrm{ps} / \mathrm{m}$ and the high-frequency band grows with $\left|C_{1}\right|$ beyond the critical value. The gain of the high-frequency band can be larger than that of the low-frequency band at high values of $C_{1}$ (Figs. 8 and 9). It is possible to change the dominant MI in terms of the frequency by increasing the total input power.

The magnitude of $C_{1 \mathrm{cr}},\left|C_{1 \mathrm{cr}}\right|$ increases with $C$ and $\left|\beta_{2}\right|$, but decreases with an increase in $P$ and $\gamma$, as shown in Fig. 10, and the patterns are different from those in the anomalous dispersion regime (Fig. 5).

\section{HIGH-ORDER EFFECTS}

It is of interest to know how robust the effects of $C_{1}$ are in the presence of high-order effects. With the third-order dispersion (TOD) and SS taken into account, Eq. (1) are generalized to
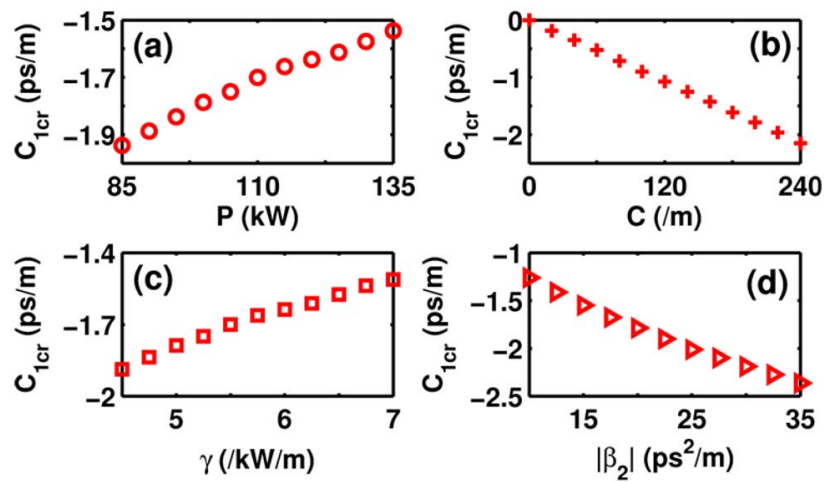

Fig. 10. (Color online) Dependence of the critical value $C_{1 \mathrm{cr}}$ on (a) total input power $P$ for $\beta_{2}=0.02 \mathrm{ps}^{2} / \mathrm{m}, \gamma=5.0 /(\mathrm{kW} \cdot \mathrm{m})$, and $C=200 / \mathrm{m}$; (b) coupling coefficient $C$ for $\beta_{2}=0.02 \mathrm{ps}^{2} / \mathrm{m}$, $\gamma=5.0 /(\mathrm{kW} \cdot \mathrm{m})$, and $P=100 \mathrm{~kW}$; (c) SPM parameter $\gamma$ for $\beta_{2}=0.02 \mathrm{ps}^{2} / \mathrm{m}, C=200 / \mathrm{m}$, and $P=100 \mathrm{~kW}$; and (d) magnitude of GVD $\left|\beta_{2}\right|$ for $\gamma=5.0 /(\mathrm{kW} \cdot \mathrm{m}), C=200 / \mathrm{m}$, and $P=100 \mathrm{~kW}$.

$$
\begin{aligned}
i \frac{\partial a_{1}}{\partial z}- & \frac{1}{2} \beta_{2} \frac{\partial^{2} a_{1}}{\partial t^{2}}-i \frac{1}{6} \beta_{3} \frac{\partial^{3} a_{1}}{\partial t^{3}}+\gamma\left|a_{1}\right|^{2} a_{1}+i \sigma \frac{\partial}{\partial t}\left(\left|a_{1}\right|^{2} a_{1}\right) \\
& +C a_{2}+i C_{1} \frac{\partial a_{2}}{\partial t}=0, \\
i \frac{\partial a_{2}}{\partial z}- & \frac{1}{2} \beta_{2} \frac{\partial^{2} a_{2}}{\partial t^{2}}-i \frac{1}{6} \beta_{3} \frac{\partial^{3} a_{2}}{\partial t^{3}}+\gamma\left|a_{2}\right|^{2} a_{2}+i \sigma \frac{\partial}{\partial t}\left(\left|a_{2}\right|^{2} a_{2}\right) \\
& +C a_{1}+i C_{1} \frac{\partial a_{1}}{\partial t}=0
\end{aligned}
$$

where $\beta_{3}$ is the TOD coefficient and $\sigma=\gamma / \omega_{0}$ is the SS coefficient with $\omega_{0}$ being the angular optical carrier frequency.

By applying the mathematical procedure described in Section 3 , we obtain the following dispersion relation:

$$
\left[\left(K_{1}+\frac{\sqrt{2}}{2} \Omega C_{1}\right)^{2}-s_{1}\right]\left[\left(K_{1}-\frac{\sqrt{2}}{2} \Omega C_{1}\right)^{2}-s_{2}\right]=s-\sigma \Omega S,
$$

where the parameters $s_{1}, s_{2}$, and $s$ are given by Eqs. (스)-(14), respectively, and

$$
K_{1}=K-\frac{1}{6} \beta_{3} \Omega^{3}-\sigma \Omega P,
$$

$$
\begin{aligned}
S= & \left(10 \frac{C^{2}}{\gamma^{2}}-3 P^{2}\right) \sigma \Omega K_{1}^{2} \\
& +2\left(4 C^{2}-\gamma^{2} P^{2}\right)\left(\frac{P}{\gamma^{2}} \sigma^{2} \Omega^{2}+\frac{2 \beta_{2}}{\gamma} \Omega^{2}-P\right) K_{1} \\
& +\beta_{2}^{2}\left(\frac{3 C^{2}}{2 \gamma^{2}}-\frac{1}{4} P^{2}\right) \sigma \Omega^{5} \\
& -\left[\left(4 C^{2}-\gamma^{2} P^{2}\right)\left(\frac{2 C_{1}^{2}}{\gamma^{2}}-\frac{P \beta_{2}}{\gamma}\right)+\frac{2 C_{1}^{2} C^{2}}{\gamma^{2}}\right] \sigma \Omega^{3} \\
& +C^{2}\left(\frac{9 C^{2}}{\gamma^{4}}-\frac{2 P^{2}}{\gamma^{2}}\right) \sigma^{3} \Omega^{3}-\left(4 C^{2}-\gamma^{2} P^{2}\right) \frac{6 C^{2}}{\gamma^{2}} \sigma \Omega \\
& +\left(4 C^{2}-\gamma^{2} P^{2}\right) \frac{8 C C_{1}}{\gamma} \Omega .
\end{aligned}
$$

According to the above expressions, $\beta_{3}$ affects only the real part of $K$ and has no effect on the imaginary part of $K$ and hence the MI gain spectrum. This is, in fact, also the case for a single-core fiber [37, $\underline{38}]$.

The effects of SS on the MI gain spectrum are shown in Fig. 11 for the anomalous dispersion regime with $C=200 / \mathrm{m}, \quad C_{1}=-1 \mathrm{ps} / \mathrm{m}, \quad \gamma=2.5 /(\mathrm{kW} \cdot \mathrm{m}), \quad \lambda=1.5 \mu \mathrm{m}$, and $\gamma / \omega_{0}=1.99 \times 10^{-3} \mathrm{ps} /(\mathrm{kW} \cdot \mathrm{m})$, and in Fig. 12 for the normal dispersion regime with $C=200 / \mathrm{m}, C_{1}=-1 \mathrm{ps} / \mathrm{m}$, $\gamma=5.0 /(\mathrm{kW} \cdot \mathrm{m}), \quad \lambda=1.0 \mu \mathrm{m}, \quad$ and $\quad \gamma / \omega_{0}=2.65 \times$ $10^{-3} \mathrm{ps} /(\mathrm{kW} \cdot \mathrm{m})$. We can see that the effects of SS become more significant only as the total input power increases. At a low input power, SS modifies the MI gain and frequency only slightly. At a very high input power (for example, $700 \mathrm{~kW}$ ), however, SS can shift the MI bands significantly, as shown in Fig. 12. In general, apart from modifying the MI gain and shifting the MI band, SS does not generate significant new MI characteristics. The results are similar to those obtained for a single-core fiber [38]. 


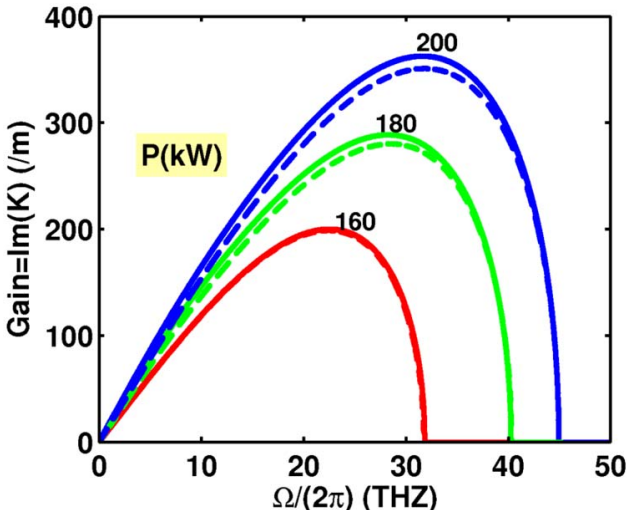

Fig. 11. (Color online) Variation of the MI gain spectrum $g(\Omega)$ with the total power $P$, calculated for the anomalous dispersion regime with $\beta_{2}=-0.02 \mathrm{ps}^{2} / \mathrm{m}, \gamma=2.5 /(\mathrm{kW} \cdot \mathrm{m}), \lambda=1.5 \mu \mathrm{m}, C=200 / \mathrm{m}$, and $C_{1}=-1 \mathrm{ps} / \mathrm{m}$ without (solid curve) and with (dashed curve) SS.

For the sake of simplicity, our MI analysis ignores stimulated Raman scattering. For a single-core fiber, stimulated Raman scattering can generate complicated MI characteristics, especially at high input power levels [39,40]. We expect similar results for a two-core fiber, given the experience with the TOD and SS. Incorporation of Raman effects in the MI analysis of a two-core fiber is a complicated problem, which deserves a detailed investigation in the future.

\section{COMPARISON WITH THE WAVE PROPAGATION ANALYSIS}

To study the evolution of MI along a two-core fiber, we solve numerically the coupled nonlinear equations Eq. (1) by launching a cw into the fiber together with a small white noise. In these simulations, we employ a pseudospectral method in the time domain and a fourth-order Runge-Kutta scheme with adaptive step-size control in the space domain [41]. The power of the added white noise is $1 \times 10^{-4} \%$ of the input $\mathrm{cw}$ power, and it covers a frequency range of $\pm 1200 \mathrm{THz}$ around the optical carrier frequency. From the theory of MI, the cw should evolve into a modulated wave as it propagates along the fiber. The onset of the modulation should occur at the dominant MI frequency, i.e., the MI frequency that has the maximum gain. The wave propagation analysis can serve as a verification of the MI analysis.

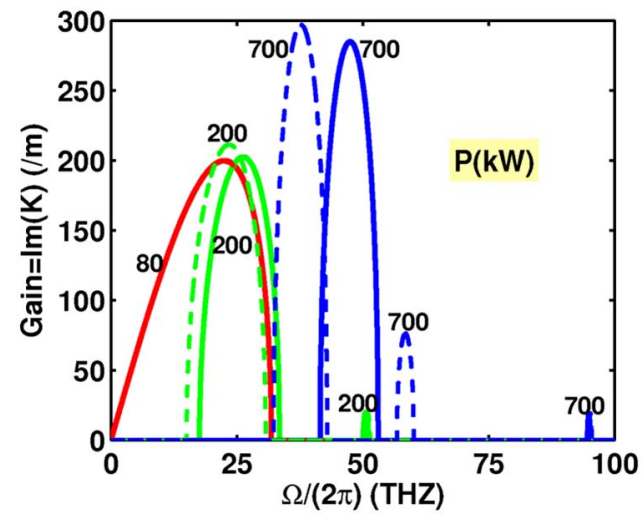

Fig. 12. (Color online) Variation of the MI gain spectrum $g(\Omega)$ with the total power $P$, calculated for the normal dispersion regime with $\beta_{2}=0.02 \mathrm{ps}^{2} / \mathrm{m}, \gamma=5.0 /(\mathrm{kW} \cdot \mathrm{m}), \lambda=1.0 \mu \mathrm{m}, C=200 / \mathrm{m}$, and $C_{1}=$ $-1 \mathrm{ps} / \mathrm{m}$ without (solid curve) and with (dashed curve) SS.
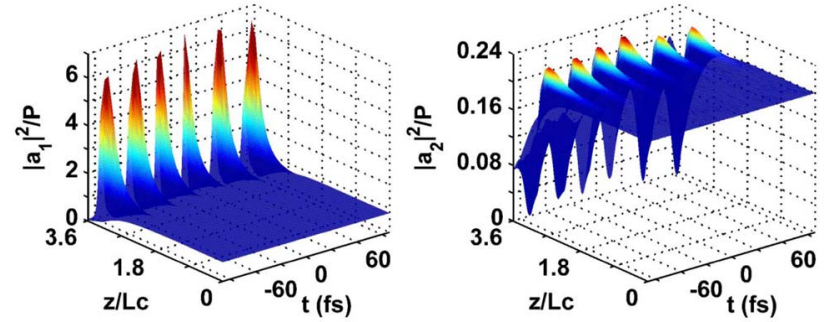

Fig. 13. (Color online) Evolution of MI from a cw input in the absence of $\operatorname{CCD}\left(C_{1}=0 \mathrm{ps} / \mathrm{m}\right)$, calculated for the anomalous dispersion regime with $\beta_{2}=-0.02 \mathrm{ps}^{2} / \mathrm{m}, \gamma=2.5 /(\mathrm{kW} \cdot \mathrm{m}), \lambda=1.5 \mu \mathrm{m}$, $C=200 / \mathrm{m}, P=200 \mathrm{~kW}$, and $P_{1} / P_{2}=4$.

We first consider the anomalous dispersion regime in the absence of $\operatorname{CCD}\left(C_{1}=0 \mathrm{ps} / \mathrm{m}\right)$. The parameters used in the simulation are $C=200 / \mathrm{m}, \beta_{2}=-0.02 \mathrm{ps}^{2} / \mathrm{m}, \gamma=2.5 /(\mathrm{kW} \cdot \mathrm{m})$, $\lambda=1.5 \mu \mathrm{m}, P=200 \mathrm{~kW}$, and $P_{1} / P_{2}=4$. Figure 13 shows the wave propagation dynamics in the two cores, where the distance is normalized with respect to the coupling length $L_{c}=\pi /(2 C)$. The cw input evolves into a modulated wave with a period of $33.4 \mathrm{fs}$ (at $z=3.2 L_{c}$ ), which corresponds to a modulation frequency of $29.9 \mathrm{THz}$. This frequency compares favorably with the dominant MI frequency, $30.4 \mathrm{THz}$, obtained from the MI analysis. We then include the effects of CCD by setting $C_{1}=-1 \mathrm{ps} / \mathrm{m}$ and show the wave propagation dynamics in Fig. 14. In this case, the cw evolves into a modulated wave with a period of $32.6 \mathrm{fs}$ (at $z=3.4 L_{c}$ ) which corresponds to a modulation frequency of $30.7 \mathrm{THz}$. The dominant MI frequency obtained from the MI analysis is $31.6 \mathrm{THz}$, in good agreement with the result from the wave propagation analysis. A comparison of Figs. 13 and 14 shows that, in the presence of CCD, it takes a longer distance for MI to occur, which agrees with the finding in Section 4.A that CCD lowers the MI gain in the anomalous dispersion regime and thus increases the distance required for the growth of the modulated wave.

We next consider the normal dispersion regime. The MI analysis predicts that, without CCD, there exist two MI bands with the high-frequency one being the predominant one, while with CCD, the low-frequency band is the predominant one, as shown in Fig. 8. Figure 15 shows the wave propagation dynamics in the two cores in the absence of CCD $\left(C_{1}=0 \mathrm{ps} / \mathrm{m}\right)$. The parameters used in the simulation are $C=200 / \mathrm{m}, \quad \beta_{2}=0.02 \mathrm{ps}^{2} / \mathrm{m}, \quad \gamma=5.0 /(\mathrm{kW} \cdot \mathrm{m}), \lambda=1.0 \mu \mathrm{m}$, $P=100 \mathrm{~kW}$, and $P_{1} / P_{2}=4$. The modulated wave evolved has a period of $32.7 \mathrm{fs}$ (at $z=8.0 L_{c}$ ), which corresponds to a modulation frequency of $30.6 \mathrm{THz}$, in excellent agreement with the dominant MI frequency in the high-frequency band $30.6 \mathrm{THz}$. Figure $\underline{16}$ shows the wave propagation dynamics
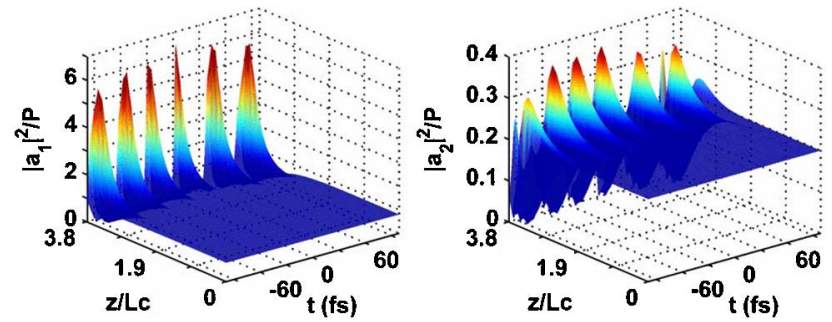

Fig. 14. (Color online) Evolution of MI from a cw input in the presence of CCD $\left(C_{1}=-1 \mathrm{ps} / \mathrm{m}\right)$, calculated for the anomalous dispersion regime with $\beta_{2}=-0.02 \mathrm{ps}^{2} / \mathrm{m}, \gamma=2.5 /(\mathrm{kW} \cdot \mathrm{m}), \lambda=1.5 \mu \mathrm{m}$, $C=200 / \mathrm{m}, P=200 \mathrm{~kW}$, and $P_{1} / P_{2}=4$. 

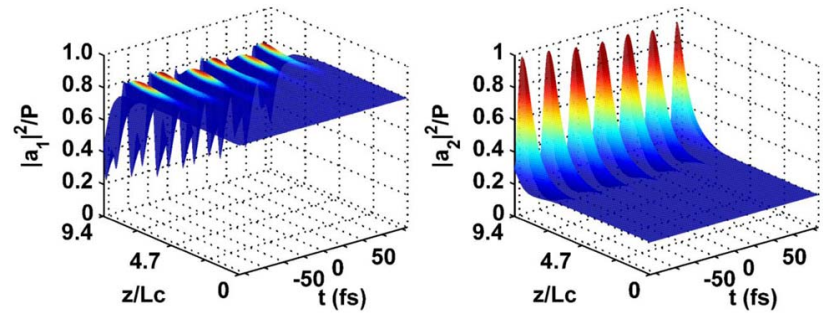

Fig. 15. (Color online) Evolution of MI from a cw input in the absence of $\operatorname{CCD}\left(C_{1}=0 \mathrm{ps} / \mathrm{m}\right)$, calculated for the normal dispersion regime with $\beta_{2}=0.02 \mathrm{ps}^{2} / \mathrm{m}, \gamma=5.0 /(\mathrm{kW} \cdot \mathrm{m}), \lambda=1.0 \mu \mathrm{m}, C=200 / \mathrm{m}$, $P=100 \mathrm{~kW}$, and $P_{1} / P_{2}=4$.
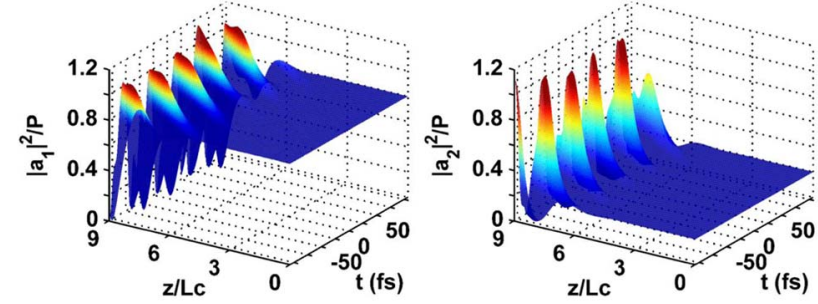

Fig. 16. (Color online) Evolution of MI from a cw input in the presence of $\operatorname{CCD}\left(C_{1}=-1 \mathrm{ps} / \mathrm{m}\right)$, calculated for the normal dispersion regime with $\beta_{2}=0.02 \mathrm{ps}^{2} / \mathrm{m}, \gamma=5.0 /(\mathrm{kW} \cdot \mathrm{m}), \lambda=1.0 \mu \mathrm{m}, C=200 / \mathrm{m}$, $P=100 \mathrm{~kW}$, and $P_{1} / P_{2}=4$.

in the two cores for $C_{1}=-1 \mathrm{ps} / \mathrm{m}$. In this case, the modulation period is $47.1 \mathrm{fs}$ (at $z=7.8 L_{c}$ ), which gives a modulation frequency of $21.2 \mathrm{THz}$, in good agreement with the dominant MI frequency of the low-frequency band $19.7 \mathrm{THz}$.

In the above examples, because the input power is much higher than the critical switching power of the two-core fiber, which is equal to $P_{c}=4 C /(\gamma \pi)=102 \mathrm{~kW}$ for the anomalous dispersion regime or $51 \mathrm{~kW}$ for the normal dispersion regime, there is little power exchange between the two cores during the evolution of MI [42]. At a sufficiently low input power, strong periodic power exchange between the two cores can be observed. It is difficult to determine the modulation frequency precisely from the wave propagation dynamics because of the quasi-periodic nature of the evolved modulated wave. To highlight the dominant modulation frequency, we display the wave propagation dynamics up to a distance that shows the onset of MI, as shown in Figs. 13-16. As the propagation distance increases, the waveform starts to lose its periodicity and becomes increasingly irregular and spiky, which implies the presence of a range of frequency components. This phenomenon is consistent with the fact that the frequency components in the MI band can grow along the fiber at different gains. The results from the MI analysis and the wave propagation analysis agree well with each other.

\section{CONCLUSIONS}

We present a detailed analysis of the MI characteristics of a two-core fiber based on solving a pair of generalized, linearly coupled nonlinear Schrödinger equations. In particular, we calculate the MI gain spectrum of the fiber in both the anomalous and normal dispersion regimes. The MI analysis agrees with the wave propagation analysis that shows explicitly the evolution of MI along the fiber from a cw input. Our study focuses mainly on the effects of the CCD, as measured by the parameter $C_{1}$ in the coupled system Eq. (1).
In general, $C_{1}$ does not affect the MI growth rate of the symmetric/antisymmetric cw state, but only changes the frequency of the MI. However, $C_{1}$ can drastically modify the MI in the asymmetric cw configuration. Roughly speaking, $C_{1}$ decreases (increases) the maximum MI growth rate in the anomalous (normal) dispersion regime, respectively. Indeed the change in maximum growth rate can be very substantial.

Another remarkable result is the existence of a critical value, $C_{1 \mathrm{cr}}$, for the $\mathrm{CCD}$, where the MI gain spectrum undergoes an abrupt change. In the anomalous dispersion regime, a new low-frequency MI band is generated at $C_{1 \mathrm{cr}}$ and the original MI band is dragged toward the high-frequency range (Figs. 3 and 4 ). In the normal dispersion regime, an MI band vanishes, reappears across $C_{1 \mathrm{cr}}$, and then moves up in frequency with increasing $\left|C_{1}\right|$, while the other MI band is enhanced in a monotonic manner (Figs. 8 and 9 ). In both dispersion regimes, the relative magnitude of the low-frequency band and the high-frequency band depends strongly on the total input power. It is possible to switch the dominant MI frequency from a low-frequency band to a high-frequency one by increasing the total input power, provided that the absolute value of $C_{1}$ is sufficiently large.

We also consider some high-order effects. In particular, we find that the TOD has no influence on the MI gain spectrum, while SS can shift the dominant MI band significantly only at a sufficiently high input power level.

Although the MI analysis is strictly valid for a cw input, the results should be valuable for a quasi-cw input, namely, pulses generated by ordinary high-power lasers. As shown by our results, the MI frequencies are located in the range from $10-100 \mathrm{THz}$ for a typical two-core fiber, which corresponds to modulated waves with periods $10-100 \mathrm{fs}$ or the generation of spectral components $80-800 \mathrm{~nm}$ away from a pump wavelength of $1550 \mathrm{~nm}$. Consequently, the MI effects should contribute to the observed generation of multiple resonance peaks in the output spectrum of a photonic crystal two-core fiber, pumped by intense $100 \mathrm{fs}$ pulses at $1550 \mathrm{~nm}$ [43]. In fact, the output spectrum calculated by numerically solving Eq. (1) for $100 \mathrm{fs}$ pulses propagating along the same fiber [44] shows resonance characteristics that are consistent with the MI analysis. The many novel features of MI obtained in our study could shed new light on the understanding of MI-related nonlinear effects in two-core fibers.

\section{ACKNOWLEDGMENTS}

Partial financial support has been provided by the Research Grants Council contract HKU7120/08E.

\section{REFERENCES}

1. C. Martijn de Sterke, "Theory of modulational instability in fiber Bragg gratings," J. Opt. Soc. Am. B 15, 2660-2667 (1998).

2. A. Parini, G. Bellanca, S. Trillo, M. Conforti, A. Locatelli, and C. De Angelis, "Self-pulsing and bistability in nonlinear Bragg gratings,” J. Opt. Soc. Am. B 24, 2229-2237 (2007).

3. G. P. Agrawal, "Modulation instability induced by cross-phase modulation," Phys. Rev. Lett. 59, 880-883 (1987).

4. T. Tanemura and K. Kikuchi, "Unified analysis of modulational instability induced by cross-phase modulation in optical fibers," J. Opt. Soc. Am. B 20, 2502-2514 (2003).

5. J. M. Chávez Boggio, S. Tenenbaum, and H. L. Fragnito, "Amplification of broadband noise pumped by two lasers in optical fibers," J. Opt. Soc. Am. B 18, 1428-1435 (2001).

6. N. C. Panoiu, X. Chen, and R. M. Osgood, "Modulation instability in silicon photonic nanowires," Opt. Lett. 31, 3609-3611 (2006). 
7. X. Dai, Y. Xiang, S. Wen, and D. Fan, "Modulation instability of copropagating light beams in nonlinear metamaterials," J. Opt. Soc. Am. B 26, 564-571 (2009).

8. C. R. Phillips and M. M. Fejer, "Stability of the singly resonant optical parametric oscillator,” J. Opt. Soc. Am. B 27, 2687-2699 (2010).

9. G. Millot, S. Pitois, and P. Tchofo Dinda, "Modulational instability processes in optical isotropic fibers under dual-frequency circular polarization pumping," J. Opt. Soc. Am. B 19, 454-460 (2002).

10. M. E. Marhic, K. K. Y. Wong, and L. G. Kazovsky, "Fiber-optical parametric amplifiers with linearly or circularly polarized waves," J. Opt. Soc. Am. B 20, 2425-2433 (2003).

11. C. J. McKinstrie, S. Radic, and C. Xie, "Parametric instabilities driven by orthogonal pump waves in birefringent fibers," Opt. Express 11, 2619-2633 (2003).

12. H. S. Chiu and K. W. Chow, "Effect of birefringence on the modulation instabilities of a system of coherently coupled nonlinear Schrödinger equations," Phys. Rev. A 79, 065803 (2009).

13. P. Tchofo Dinda and K. Porsezian, "Impact of fourth-order dispersion in the modulational instability spectra of wave propagation in glass fibers with saturable nonlinearity,” J. Opt. Soc. Am. B 27, 1143-1152 (2010).

14. E. A. Ultanir, D. N. Christodoulides, G. I. Stegeman, "Spatial modulation instability in periodically patterned semiconductor optical amplifiers,” J. Opt. Soc. Am. B 23, 341-346 (2006).

15. J. M. Dudley, G. Genty, F. Dias, B. Kibler, and N. Akhmediev, "Modulation instability, Akhmediev Breathers, and continuous wave supercontinuum generation," Opt. Express 17, 21497-21508 (2009).

16. Y. S. Kivshar and G. P. Agrawal, Optical Solitons: From Fibers to Photonic Crystals (Academic, 2003).

17. A. Höök and M. Karlsson, "Ultrashort solitons at the minimumdispersion wavelength: effect of fourth-order dispersion," Opt. Lett. 18, 1388-1390 (1993).

18. T. Tanemura, Y. Ozeki, and K. Kikuchi, "Modulational instability and parametric amplification induced by loss dispersion in optical fibers," Phys. Rev. Lett. 93, 163902 (2004).

19. C. Cambournac, H. Maillotte, E. Lantz, J. M. Dudley, and M. Chauvet, "Spatiotemporal behavior of periodic arrays of spatial solitons in a planar waveguide with relaxing Kerr nonlinearity," J. Opt. Soc. Am. B 19, 574-585 (2002).

20. G. V. Simaeys, P. Emplit, and M. Haelterman, "Experimental study of the reversible behavior of modulational instability in optical fibers," J. Opt. Soc. Am. B 19, 477-486 (2002)

21. J. Wang, L. Li, Z. Li, G. Zhou, D. Mihalache, and B. A. Malomed, "Generation, compression, and propagation of pulse trains under higher order effects," Opt. Commun. 263, 328-336 (2006).

22. A. W. Snyder, "Coupled-mode theory for optical fibers," J. Opt. Soc. Am. 62, 1267-1277 (1972).

23. K.-I. Kitayama and Y. Ishida, "Wavelength-selective coupling of two-core optical fiber: application and design,” J. Opt. Soc. Am. A 2, 90-94 (1985).

24. K. S. Chiang, "Propagation of short optical pulses in directional couplers with Kerr nonlinearity," J. Opt. Soc. Am. B 14, 1437-1443 (1997).

25. K. S. Chiang, "Coupled-mode equations for pulse switching in parallel waveguides,” IEEE J. Quantum Electron. 33, 950-954 (1997).
26. P. Shum, K. S. Chiang, and W. A. Gambling, "Switching dynamics of short optical pulses in a nonlinear directional coupler," IEEE J. Quantum Electron. 35, 79-83 (1999).

27. P. M. Ramos and C. R. Paiva, "All-optical pulse switching in twincore fiber couplers with intermodal dispersion," IEEE J. Quantum Electron. 35, 983-989 (1999).

28. V. Rastogi, K. S. Chiang, and N. N. Akhmediev, "Soliton states in a nonlinear directional coupler with intermodal dispersion," Phys. Lett. A 301, 27-34 (2002).

29. S. C. Tsang, K. S. Chiang, and K. W. Chow, "Soliton interaction in a two-core optical fiber," Opt. Commun. 229, 431-439 (2004).

30. K. S. Chiang, Y. T. Chow, D. J. Richardson, D. Taverner, L. Dong, L. Reekie, and K. M. Lo, "Experimental demonstration of intermodal dispersion in a two-core optical fiber," Opt. Commun. 143, 189-192 (1997)

31. P. Peterka, P. Honzatko, J. Kanka, V. Matejec, and I. Kasik, "Generation of high-repetition rate pulse trains in a fiber laser through a twin-core fiber," Proc. SPIE 5036, 376-381 (2003).

32. S. Trillo, S. Wabnitz, G. I. Stegeman, and E. M. Wright, "Parametric amplification and modulation instabilities in dispersive nonlinear directional couplers with relaxing nonlinearity," J. Opt. Soc. Am. B 6, 889-900 (1989).

33. R. S. Tasgal and B. A. Malomed, "Modulational instabilities in the dual-core nonlinear optical fiber," Phys. Scr. 60, 418-422 (1999).

34. K. S. Chiang, "Intermodal dispersion in two-core optical fibers," Opt. Lett. 20, 997-999 (1995)

35. Z. Wang, T. Taru, T. A. Birks, J. C. Knight, Y. Liu, and J. Du, “Coupling in dual-core photonic bandgap fibers: theory and experiment," Opt. Express 15, 4795-4803 (2007).

36. M. Liu and K. S. Chiang, "Pulse propagation in a decoupled twocore fiber," Opt. Express 18, 21261-21268 (2010).

37. S. C. Wen and D. Y. Fan, "Spatiotemporal instabilities in nonlinear Kerr media in the presence of arbitrary higher-order dispersions," J. Opt. Soc. Am. B 19, 1653-1659 (2002).

38. W. C. Xu, S. M. Zhang, W. C. Chen, A. P. Luo, and S. H. Liu, "Modulation instability of femtosecond pulses in dispersiondecreasing fibers," Opt. Commun. 199, 355-360 (2001).

39. I. M. Uzunov, "Influence of intrapulse Raman scattering on the modulational instability in optical fibers," Opt. Quantum Electron. 22, 529-533 (1990).

40. G. Millot, P. Tchofo Dinda, E. Seve, and S. Wabnitz, "Modulational instability and stimulated Raman scattering in normally dispersive highly birefringent fibers," Opt. Fiber Technol. 7, 170-205 (2001).

41. L. D. Carr, J. N. Kutz, and W. P. Reinhardt, "Stability of stationary states in the cubic nonlinear Schrodinger equation: applications to the Bose-Einstein condensate," Phys. Rev. E 63, 066604 (2001).

42. S. M. Jensen, "The nonlinear coherent coupler," IEEE J. Quantum Electron. 18, 1580-1583 (1982).

43. A. Betlej, S. Suntsov, K. G. Makris, L. Jankovic, D. N Christodoulides, G. I. Stegeman, J. Fini, R. T. Bise, and D. J. DiGiovanni, "All-optical switching and multifrequency generation in a dual-core photonic crystal fiber," Opt. Lett. 31, 1480-1482 (2006).

44. M. Liu and K. S. Chiang, "Propagation of ultrashort pulses in a nonlinear two-core photonic crystal fiber," Appl. Phys. B 98, 815-820 (2009). 